\title{
Análisis del sistema implantado en España para el control de equipos de aplicación de productos fitosanitarios: entidades inspectoras, control de la calidad de la inspección y gestión de datos
}

\author{
A. Jiménez ${ }^{1}$, M. Vidal ${ }^{1}$, A. Boné ${ }^{1}$, F.J. García Ramos ${ }^{1}$ \\ 1 Escuela Politécnica Superior, Ctra. Cuarte s/n, 22071 Huesca; angelji@unizar.es; vidalcor@unizar.es; \\ anbone@unizar.es; fjavier@unizar.es
}

\begin{abstract}
Resumen: El Real Decreto 1702/2011, de 18 de noviembre, de inspecciones periódicas de los equipos de aplicación de productos fitosanitarios, establece que las CCAA son las encargadas del "control y aplicación del programa de inspecciones que se lleven a cabo en su ámbito territorial", para dar cumplimiento a la obligación que la Directiva 2009/128/CE de uso sostenible de plaguicidas impuso a todos los países miembros de la UE. Para poder llevar a cabo dicha encomienda la mayoría de las CCAA (trece) promulgaron decretos u órdenes autonómicos, donde se regula lo relativo al establecimiento y funcionamiento del sistema necesario para poder realizar la inspección periódica de los equipos de aplicación de fitosanitarios (EAF), desde el proceso de autorización de las entidades inspectoras (ITEAF) hasta los mecanismos de control de la calidad de la propia actividad inspectora y de los procesos relativos a la gestión de los resultados de las inspecciones realizadas en su ámbito territorial. El resto de las autonomías, cuyo número de equipos a inspeccionar es muy reducido, decidieron que fuesen los servicios del Departamento competente los que se encargaran de realizar la inspección de las máquinas y las instalaciones. En este artículo se analiza la configuración actual del sistema completo, en el que están implicadas las ITEAF autorizadas, los Órganos Competentes de las CCAA y la Dirección General de Medios de Producción del MAPA. Se describe el modelo establecido y las herramientas con las que se ha dotado a los agentes implicados. Asimismo, se determinan las fortalezas y debilidades del sistema de gestión, una vez que se encuentra en la fase final del proceso de implantación del Plan Nacional de inspección obligatoria de todos los equipos de aplicación de productos fitosanitarios, independientemente de que la aplicación se realice con equipos móviles o aeronaves a cultivos en campo abierto, con equipos fijos y semimóviles en el interior de instalaciones para cultivos bajo cubierta o mediante equipos fijos.
\end{abstract}

Palabras clave: pulverizador, ITEAF, plaguicida, manual de inspección, APPRITEAF.

\section{Introducción}

La Ley de sanidad vegetal, 43/2002, de 20 de noviembre, y la Directiva 2009/128/CE, de 21 de octubre, del Parlamento Europeo y del Consejo, por la que se establece un uso sostenible de los plaguicidas, determinan los requisitos aplicables al uso de los plaguicidas y de los medios técnicos que se emplean para su aplicación.

Uno de los aspectos que integran el Plan Nacional es la puesta en marcha de la inspección técnica obligatoria de los equipos de aplicación, con el objetivo de contribuir a la protección 


\section{CONGRESO IBÉRICO DE AGROINGENIERÍA \\ X CONGRESSO IBÉRICO DE AGROENGENHARIA \\ 3 - 6 septiembre 2019, Huesca - España}

medioambiental y a la seguridad de los aplicadores, y por ello, el Ministerio de Agricultura, Pesca y Alimentación (MAPA) publicó el Real Decreto 1702/2011, de 18 de noviembre, sobre las inspecciones obligatorias de los equipos de aplicación de productos fitosanitarios.

El artículo 5.1 obliga a que todos los EAF estén inscritos, en el ROMA o los Censos auxiliares habilitados, y en el artículo 5.2 indica que se deben establecer los programas de inspección para que todos los equipos en uso estén inspeccionados, al menos una vez, antes del 26 de noviembre de 2016 (los equipos nuevos no están obligados hasta que no cumplan cinco años), tal y como impone la Directiva 2009/128/CE.

En la tabla 1 se muestra la situación actual de los equipos inspeccionados, clasificados por tipologías, y los equipos que, estando censados, no han sido inspeccionados ninguna vez. No están recogidos los equipos no censados, ni los equipos que se han censado como nuevos y todavía están dentro de sus primeros cinco años, y por lo tanto no tienen obligación de ser inspeccionados. Tampoco se reflejan las máquinas censadas y que se corresponden con tipologías exentas de inspección (mochilas y carretillas manuales).

Tabla 1. Situación a 10/05/2019 del censo de equipos a inspeccionar. Fuente: REGITEAF (MAPA)

\begin{tabular}{|c|c|c|c|c|c|c|c|}
\hline $\begin{array}{c}\text { Comunidad } \\
\text { Autónoma }\end{array}$ & Agrícola & $\begin{array}{c}\text { No } \\
\text { agrícola }\end{array}$ & Invernaderos & $\begin{array}{c}\text { I. } \\
\text { permanentes } \\
\end{array}$ & Aeronaves & Total & $\begin{array}{c}\text { No } \\
\text { inspeccionados } \\
\end{array}$ \\
\hline Andalucia & 73.501 & 222 & 8.650 & 1 & 38 & 82.412 & 35.858 \\
\hline Aragón & 15.975 & 32 & 0 & 5 & 0 & 16.012 & 5.165 \\
\hline Canarias & 915 & 80 & 3.611 & 0 & 0 & 4.606 & 2.969 \\
\hline Cantabria & 144 & 5 & 0 & 0 & 0 & 149 & 79 \\
\hline $\begin{array}{l}\text { Castilla-La } \\
\text { Mancha }\end{array}$ & 36.474 & 79 & 13 & 138 & 0 & 36.704 & 12.514 \\
\hline Castilla y León & 30.897 & 80 & 1 & 3 & 0 & 30.981 & 13.756 \\
\hline Cataluña & 21.716 & 224 & 2 & 1 & 5 & 21.948 & 5.618 \\
\hline $\begin{array}{l}\text { Comunidad de } \\
\text { Madrid }\end{array}$ & 1.651 & 293 & 0 & 0 & 0 & 1.944 & 883 \\
\hline $\begin{array}{l}\text { Comunidad } \\
\text { Foral de Navarra }\end{array}$ & 4.653 & 1 & 0 & 12 & 0 & 4.666 & 1.392 \\
\hline $\begin{array}{l}\text { Comunidad } \\
\text { Valenciana }\end{array}$ & 21.518 & 224 & 5 & 850 & 0 & 22.597 & 8.305 \\
\hline Extremadura & 13.268 & 5 & 8 & 0 & 0 & 13.281 & 5.513 \\
\hline Galicia & 14.793 & 15 & 0 & 0 & 1 & 14.809 & 7.011 \\
\hline Islas Baleares & 993 & 2 & 0 & 0 & 0 & 995 & 530 \\
\hline La Rioja & 7.924 & 1 & 0 & 0 & 0 & 7.925 & 2.188 \\
\hline País Vasco & 3.077 & 16 & 0 & 0 & 0 & 3.093 & 1.283 \\
\hline $\begin{array}{c}\text { Principado de } \\
\text { Asturias }\end{array}$ & 622 & 19 & 0 & 0 & 0 & 641 & 296 \\
\hline $\begin{array}{c}\text { Región de } \\
\text { Murcia } \\
\end{array}$ & 9.890 & 102 & 32 & 1 & 2 & 10.027 & 3.689 \\
\hline TOTAL & 258.011 & 1.400 & 12.322 & 1.011 & 46 & 272.790 & 107.049 \\
\hline
\end{tabular}

OBSERVACIONES: "NO INSPECCIONADOS", con este concepto englobamos los equipos inscritos en el Registro Oficial de Maquinaria Agrícola (ROMA), que estando dados de alta en el mismo, no se han inspeccionado en los últimos cinco años. 


\section{CONGRESO IBÉRICO DE AGROINGENIERÍA \\ X CONGRESSO IBÉRICO DE AGROENGENHARIA \\ 3- 6 septiembre 2019, Huesca - España}

El mencionado Real Decreto establece también que el sistema de inspección de los equipos de aplicación de productos fitosanitarios (EAF), estará estructurado en tres niveles, tal y como se muestra en la figura 1. Las Entidades Inspectoras (ITEAF), integradas por una o varias Unidades Inspectoras, son las encargadas de realizar las inspecciones, previa autorización del Órgano Competente correspondiente a la Comunidad Autónoma donde tenga su domicilio social. Los órganos competentes serán los encargados de recoger y auditar las inspecciones realizadas a las máquinas anotadas en su registro oficial de maquinaria agrícola y en sus censos auxiliares. Estos órganos serán los encargados de transmitir a la base estatal de inspecciones (REGITEAF), gestionada por el MAPA.

Este proceso se implementó con modelos diferentes y a ritmos desiguales en las diferentes Comunidades Autónomas, como consecuencia de las diferencias en cuanto a las tipologías de EAFs censadas y a las diferentes interpretaciones del RD 1702/2011.

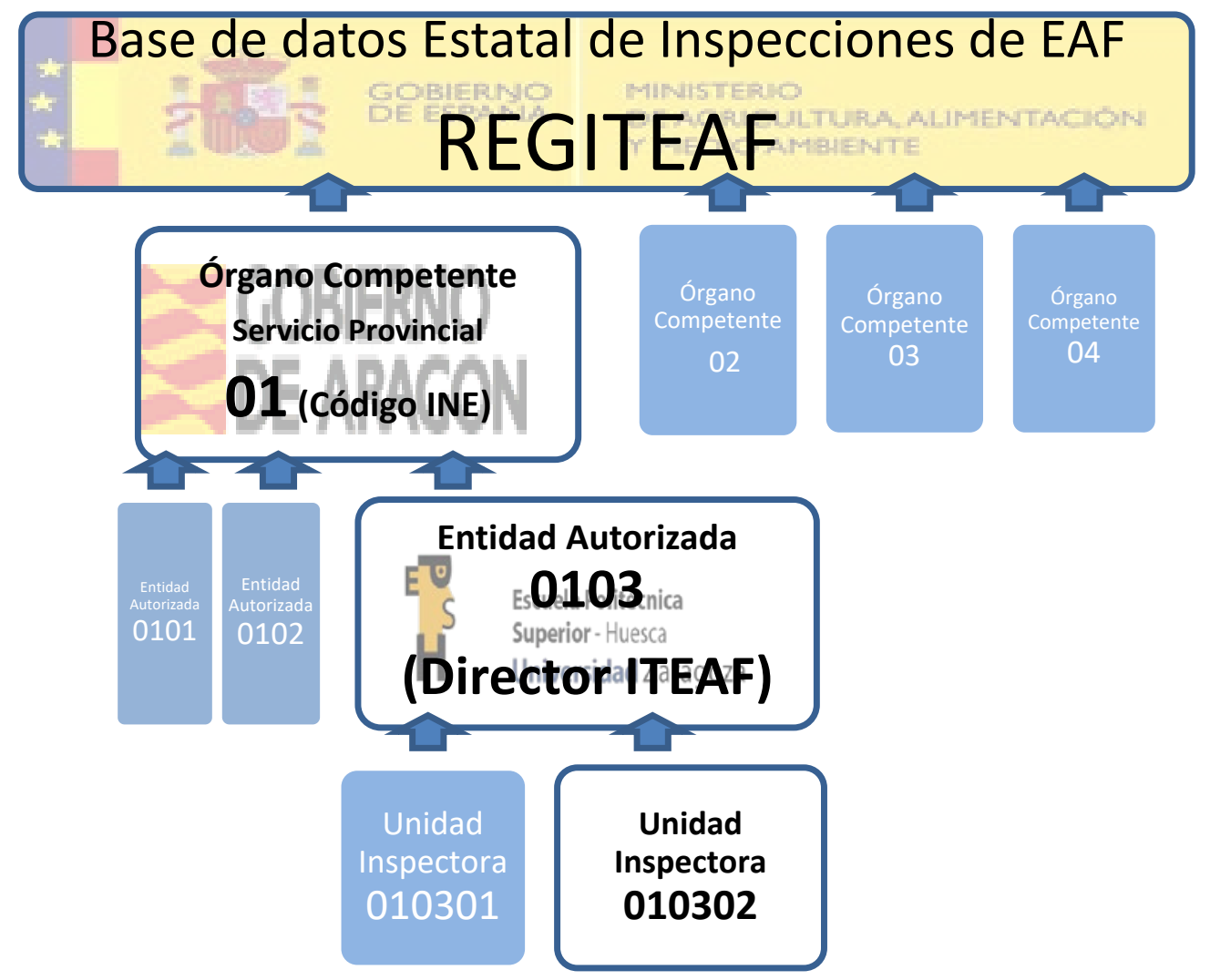

Figura 1. Estructura de la inspección de EAF en España. (Elaboración propia)

\section{Gestión de la Inspección de equipos de aplicación de productos fitosanitarios en España}

En el mencionado Real Decreto de inspecciones periódicas de los equipos de aplicación de productos fitosanitarios se detallan los requisitos exigibles a las entidades que tienen acceso a la autorización administrativa para poder realizar las inspecciones y adquirir la condición de ITEAF, así como el equipamiento y cualificación mínimos con que deberá desarrollar su actividad.

Un aspecto diferenciador, con los sistemas establecidos en otros países de la Unión Europea, es la restricción, establecida en el artículo 7, respecto a la titularidad de las ITEAF, puesto que las empresas dedicadas a la fabricación o reparación de EAF no podrán ser titulares ni tener participación en ellas, a no ser que el órgano competente lo considere imprescindible, para garantizar el servicio. Esto ha supuesto que, hasta el momento, los concesionarios y talleres 


\section{CONGRESO IBÉRICO DE AGROINGENIERÍA \\ X CONGRESSO IBÉRICO DE AGROENGENHARIA \\ 3 - 6 septiembre 2019, Huesca - España}

dedicados a la venta y reparación de equipos no han sido autorizados y las que están operando se han establecido a título personal o asociado, como secciones de empresas existentes dedicadas a prestar servicios al sector agrario o venta de materias primas, cooperativas y organizaciones de productores agrarios, centros tecnológicos públicos y privados. En Cantabria, Islas Baleares y Bizkaia, dado que el número de equipos censados es muy bajo, el servicio se presta desde la propia administración.

En cualquier caso, el RD dispone que serán los Órganos Competentes de cada comunidad autónoma los responsables de conceder la pertinente autorización, así como de realizar el control de la actividad que realicen las ITEAFs dentro de su demarcación territorial. Para poder realizar esa labor, doce de las diecisiete administraciones autonómicas y la administración Foral de Bizkaia, han recurrido a Órdenes autonómicas y Foral o Decretos autonómicos, donde se regulan las condiciones en las que se deben prestar las inspecciones periódicas de los EAF, en sus respectivos territorios. Tan solo Cantabria, Islas Baleares, Islas Canarias, Comunidad de Madrid y los Administraciones Forales de Álava y Guipúzcoa, no han considerado necesario el promulgar una regulación específica, por lo que se rigen por lo dispuesto en el RD 1702/2011.

Con todo ello, desde que, a finales de 2012, se registrara la primera autorización administrativa, se han ido concediendo autorizaciones y alguna baja. A 10 de mayo de 2019, permanecen autorizadas un total de 163 ITEAFs, y su distribución por comunidades autónomas se muestra en la figura 2.

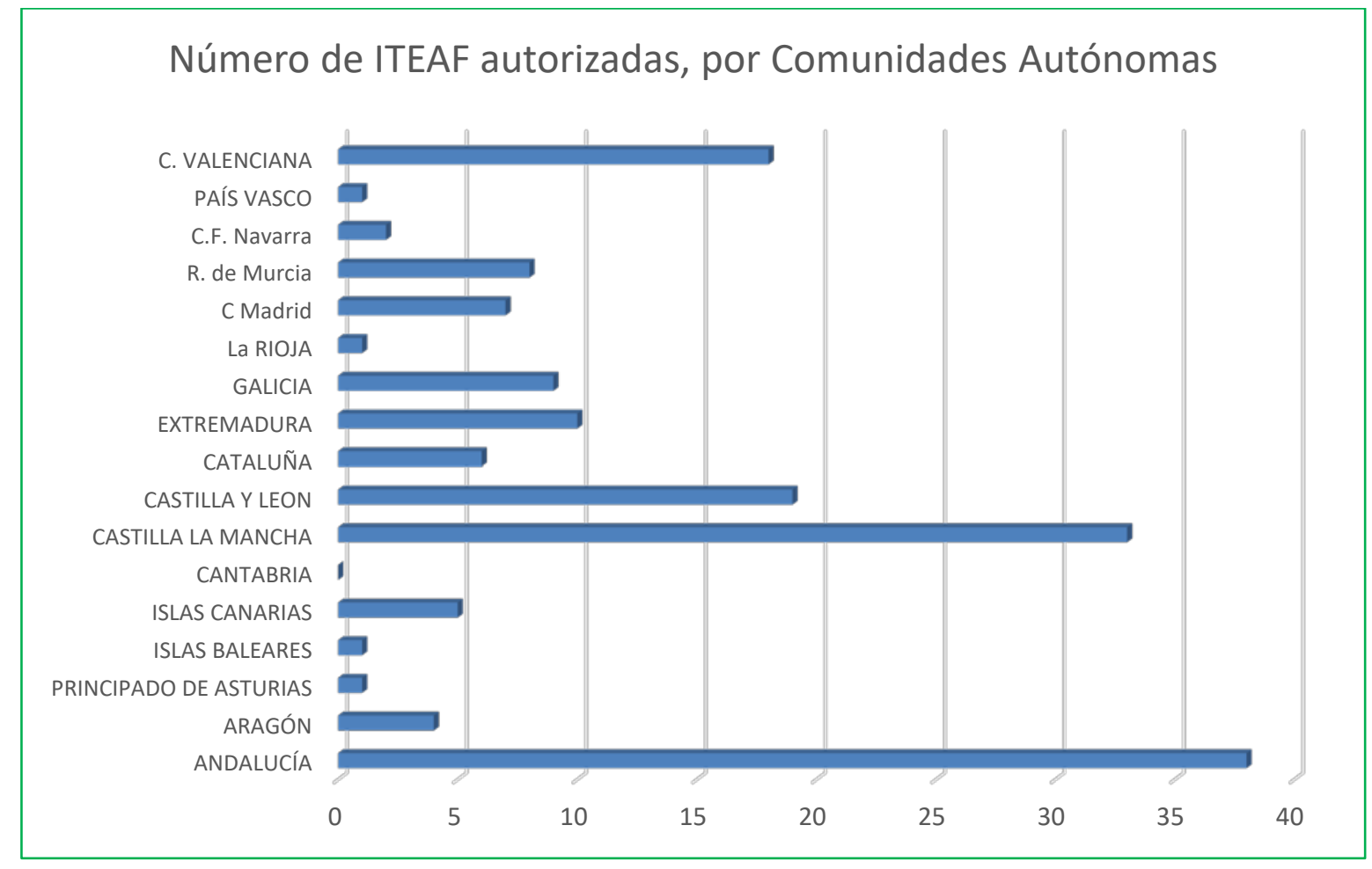

Figura 2. Número de ITEAF autorizadas por comunidad autónoma (Elaboración propia a partir de datos del MAPA a 10/05/2019).

Hay que aclarar que la autorización de una ITEAF, si bien la concede el órgano competente de una comunidad autónoma para trabajar dentro de su territorio, tiene validez en el resto del Estado español, si bien necesita comunicar la intención de trabajar en otra demarcación y deberá cumplir lo que la regulación e instrucciones locales exijan, en aquellas autonomías que tengan regulación propia. Por esta razón el número de ITEAFs a disposición de los titulares puede ser superior a los que aparecen en la figura 2. 


\section{CONGRESO IBÉRICO DE AGROINGENIERÍA \\ X CONGRESSO IBÉRICO DE AGROENGENHARIA \\ 3 - 6 septiembre 2019, Huesca - España}

\section{Gestión de los resultados de las inspecciones}

En el artículo 12 del RD 1702/2011, se especifica la documentación que las estaciones ITEAF deberán emitir y su contenido mínimo. También se indica que el Director de la ITEAF deberá remitir, al órgano competente de la comunidad autónoma donde esté inspeccionando, un documento informático con los resultados de las inspecciones. El formato y contenido de esos ficheros ha sido acorado entre el MAPA y las CCAA y está concretado en una instrucción, con el fin de que la información recibida en los órganos competentes esté normalizada y pueda ser revisada y cargada en el repositorio estatal REGITEAF, tal y como se ha representado en la figura 1 , con el fin de que dicha base de datos sea el lugar donde todas las administraciones del estado puedan consultar la información referente a los equipos de aplicación de fitosanitarios registrados.

Desde el establecimiento del sistema de inscripción e inspección obligatorias de los EAF en España, fue implantado un modelo consistente en la publicación de manuales de inspección y una herramienta informática que desarrolló protocolos de inspección por tipologías de máquinas de aplicación. Esta importante labor, promovida en el seno del Grupo de Trabajo de Técnicas y Equipos de Aplicación de Fitosanitarios, dependiente de la Subdirección General de Medios de Producción Agrícolas y Oficina Española de Variedades Vegetales. Los manuales, que facilitan la interpretación de las normas de inspección y facilitan la ejecución de la misma, han sido elaborados por Departamentos de las Escuelas de Ingenieros Agrónomos de la Universidad Politécnica de Cataluña, la Universidad de Córdoba, la Universidad Politécnica de Valencia, la Universidad de Castilla La Mancha y la Universidad de León. La aplicación informática empleada para la toma de datos, la expedición de la documentación de las inspecciones y la elaboración y transmisión de los ficheros normalizados desde las ITEAF a los órganos competentes fue desarrollada y mantenida por el Laboratorio de Maquinaria Agrícola de la Escuela Politécnica Superior de Huesca (Universidad de Zaragoza), quien, con el apoyo económico del MAPA, ha puesto a disposición de las entidades inspectoras y los órganos competentes las aplicaciones PRITEAF y APPRITEAF, facilitando el trabajo y contribuyendo a la uniformización de los procesos de toma de datos y gestión de los resultados de las inspecciones.

A pesar de que el sistema de gestión que hemos descrito es único en Europa y ha estado coordinado por el MAPA y su Laboratorio Nacional de Referencia, tiene una cadena de trasmisión de la información que podríamos denominar "manual", de tal forma que el registro de una inspección en REGITEAF depende de que el Inspector de la unidad inspectora trasmita el resultado de cada inspección realizada al Director de su ITEAF, quien, después de revisarla, lo comunica al Órgano Competente, para que el funcionario al cargo de esa labor, cargue en la base de datos estatal los registros. Este proceso es notoriamente mejorable, con el fin de reducir el trabajo que supone y, sobre todo, evitar que la información pueda perderse en el camino. Para el aseguramiento de la calidad del proceso se hace necesaria una estructura de control en cada órgano competente.

\section{Control de la actividad inspectora}

Tal y como se expresa en el Artículo 6.1 del RD 1702/2011, son las comunidades autónomas las encargadas de definir y proponer el programa de inspecciones para su territorio. En las CCAA que no prestan directamente el servicio, dicho programa incluye las labores del control de las entidades inspectoras autorizadas para inspeccionar en su territorio.

Para llevar a cabo esta encomienda, en España, nos podemos encontrar con dos modelos:

a) Personal propio de la administración (funcionarios y/o contratados).

b) Externalización parcial, mediante la contratación de un Departamento universitario o un Centro tecnológico, que proporcione la asistencia técnica al personal funcionario del órgano competente. 


\section{CONGRESO IBÉRICO DE AGROINGENIERÍA \\ X CONGRESSO IBÉRICO DE AGROENGENHARIA \\ 3 - 6 septiembre 2019, Huesca - España}

El aseguramiento de la calidad, del sistema de inspección de EAF, lo podríamos sintetizar en tres labores:

1. Vigilancia de que las entidades inspectoras siguen cumpliendo los requisitos exigidos para su autorización. Fundamentalmente se concretan en la adecuada formación de su personal y el correcto estado del equipamiento técnico, tanto material como inmaterial.

2. Control documental de las inspecciones realizadas por cada ITEAF, con la comprobación del cumplimiento de los planes de inspección autonómicos y la correspondencia de cada inspección con los registros de inscripción de las máquinas.

3. La verificación en campo de que a las máquinas inspeccionadas se les está aplicando las exigencias reflejadas en los manuales o guías de inspección publicados en la web del MAPA.

En varias de las comunidades autónomas, el elevado número de entidades autorizadas (figura 2) está dificultando que se puedan realizar adecuadamente las labores descritas, en particular la tercera de ellas, puesto que supone tener, o contratar, personal técnico y equipamiento, que se tendrá que desplazar por todo el territorio y realizar una labor de muestreo aleatorio. Para que este control sea efectivo se debe realizar de forma sorpresiva y garantizando la calidad técnica del equipamiento y la cualificación del personal que la realiza.

Estas tareas no fueron tenidas en cuenta, ni en la dotación de personal ni presupuestariamente, cuando se designaron los órganos competentes en cada Autonomía y por esa razón la mayor parte de ellos se están encontrando con grandes dificultades para poder realizar sus funciones.

Especial dificultad están teniendo los órganos competentes para aplicar sanciones a las ITEAF, que tras la verificación del incumplimiento de sus funciones, deben ser expedientadas y suspendidas de sus funciones, de forma definitiva o temporalmente. Si repasamos las órdenes y decretos autonómicos que regulan la implantación y aplicación de los planes de inspección, no encontramos herramientas específicas que les permitan aplicar un proceso sancionador más específico y adecuado a esta modalidad de autorización administrativa. Los expedientes que se han practicado en algunas CCAA, y que han terminado en la desautorización de la ITEAF infractora, han sido escasos y han tenido que superar un proceso excesivamente complejo y laborioso, sobre todo si se compara con las facilidades que tuvieron para la concesión de la autorización

\section{Conclusiones y recomendaciones}

El cumplimiento de lo dispuesto en la Directiva 2009/128/CE de uso sostenible de plaguicidas en España se ha llevado a cabo mediante la publicación del RD 1702/2011 y las correspondientes Ordenes o Decretos Autonómicos que regulan y organizan un sistema obligatorio de inspecciones de los equipos de aplicación de fitosanitarios, para lo que se ha establecido un sistema de autorización administrativa a Entidades Inspectoras, de carácter privado en su mayoría, con ámbito de actuación estatal.

Para garantizar la calidad y uniformidad de las inspecciones técnicas, se ha promovido y coordinado desde el MAPA la elaboración y publicación en su web de una serie de manuales y guías que interpretan las normas técnicas aplicables y uniformizan la actuación de los inspectores. Complementariamente a esos manuales y guías, se ha desarrollado una aplicación informática que tiene implementados los protocolos de inspección específicos para cada tipo de equipo, facilitando la toma de datos, la cumplimentación de la documentación y la generación de los datos relativos a cada inspección.

La gestión del plan de inspecciones obligatorias y el control de la calidad de las propias inspecciones técnicas está en manos de los Órganos Competentes de las CCAA, quienes lo ejercen, en la mayor parte de los casos, con unos medios propios, materiales y humanos, muy 


\section{CONGRESO IBÉRICO DE AGROINGENIERÍA \\ X CONGRESSO IBÉRICO DE AGROENGENHARIA \\ 3 - 6 septiembre 2019, Huesca - España}

limitados y que es insuficiente en aquellas autonomías donde se ha dejado en manos de entidades privadas una actividad inspectora que no ha sido, por lo general, bien recibida por los titulares de los equipos y que por lo tanto es sensible a las presiones propias de la competencia empresarial.

La gestión de la información relativa a los equipos está contenida en dos bases de datos que recogen la inscripción de los EAF, denominadas REGMAQ y REGANIF, y una base de datos, REGITEAF, que registra los resultados de las inspecciones realizadas a aquellos equipos inscritos. Las primeras son nutridas por los registros provinciales de maquinaria agrícola y los servicios de gestión de las instalaciones, mientras que los resultados de las inspecciones son subidos por los órganos competentes. Las bases de datos nombradas pertenecen a los servicios informáticos del MAPA, y por lo tanto están conectadas, pero la información relativa a los resultados de las inspecciones, proceden de la transmisión manual desde las entidades inspectoras que, como hemos dicho, son mayoritariamente privadas. El establecimiento de un sistema que integrase, en los mismos servicios ministeriales que gestionan las bases de datos, las herramientas informáticas para la realización de las inspecciones, simplificaría el manejo y aseguraría el procesamiento y almacenamiento de los datos. 